\title{
LA UE: \\ UN PROYECTO INSTITUCIONAL GLOBAL
}

Por: Marybel Pérez Suárez*

\section{Resumen}

A lo largo de sus cincuenta años de existencia, la Unión Europea cuenta, entre sus vivencias, con varios procesos de ampliación y profundización, todos ellos destinados de forma particular a consolidar para sus ciudadanos un entorno libre, seguro y próspero en el cual desenvolverse. Con este objetivo ha desarrollado una visión particular sobre temas tan relevantes como la globalización, el medioambiente, la democracia y el gobierno internacional, que marcan su agenda internacional $y$, en algunos casos, le caracterizan como un actor cosmopolita. Desde esta perspectiva, este trabajo tiene como objetivo realizar un breve balance sobre el transcurso del modelo europeo desde su constitución y perfilar sus perspectivas a medio plazo. El modelo será estudiado utilizando una perspectiva teórica política. Las perspectivas serán analizadas de dos formas: primero, observando la Unión Europea como actor cosmopolita en el sistema internacional $y$, segundo, considerando las implicaciones que esto tendrá en las relaciones con América Latina y con Colombia específicamente.

\section{Palabras clave}

Unión Europea, América Latina, Colombia, interdependencia, tendencias, globalización, seguridad, democracia, cosmopolitismo.

\section{INTRODUCCIÓN}

D esde el final de la Guerra Fría, vienen consolidándose en el mundo tendencias que confluyen en la intensificación de la interdependencia entre los diversos actores de la sociedad internacional. La multipolaridad, la globalización, los procesos multilaterales, la situación medioambiental, las migraciones y la cada vez más intensa movilización de la sociedad civil fuera de las fronteras nacionales son, entre otros, fenómenos que están transformando el sistema internacional y hacen de la interdependencia la palabra clave que le describe.

Esta interdependencia está generando modelos de convivencia en diversas regiones del mundo que en algunos casos, como el Europeo, parece acercase en determinados aspectos al cosmopolitismo. En la celebración de su cincuenta aniversario, el mensaje de la Unión Europea se ha enfocado en demostrar que a lo largo de este tiempo se han realizado

\footnotetext{
* Licenciada en Ciencias Políticas y Doctoranda en Relaciones Internacionales. Investigadora del Grupo Prospectiva y Análisis Económico Internacional.
} 
grandes avances a favor de sus ciudadanos: un espacio con libertad de movimiento, de garantías para el consumidor, que trabaja a favor de la sostenibilidad para el bienestar y que ofrece oportunidades a sus ciudadanos. También se muestra como una unidad ambiciosa sobre el orden internacional al impulsar en todos los ámbitos su compromiso con la democracia, los derechos humanos, el desarrollo y la paz.

El cosmopolitismo en términos generales persigue establecer un orden mundial de paz que favorezca el bienestar de los ciudadanos por encima de las fronteras ${ }^{1}$. Este bienestar se trata de la consolidación de la libertad y la igualdad, que actualmente, en occidente, se concibe alcanzar en un marco democrático y de libre mercado que proteja a los consumidores. Desde este punto de vista, la Unión Europea es un espacio supranacional que en cierta medida tiende al cosmopolitismo y lo promueve.

Tras cincuenta años en un proceso que probablemente ha cristalizado de un modo no esperado en sus inicios y tras un reciente, intenso y exitoso paso hacia el fortalecimiento del modelo, que se ha materializado a través de las estrategias de ampliación y profundización, existe una gran preocupación sobre el futuro de la Unión. Pese a la reciente aprobación del tratado constitucional, su rechazo en el año 2005 por parte de franceses $y$ holandeses ha permitido a algún constitucionalista, a euroescépticos y a algunos otros analistas suspicaces, mantener abierto el debate sobre las capacidades de la UE para no perder el ritmo y seguir avanzando. Este ánimo nos desafía a seguir buscando opciones para la futura evolución de la Unión y que esta sea cada vez más capaz de ofrecer garantías a sus ciudadanos y a los del resto

1 No es un modelo que pretende sustituir al Estado, sino ampliar la cantidad de canales a través de los cuales se puede establecer un orden justo para los ciudadanos.

\section{A bstract}

Throughout its fifty years of history, the European Union has implemented different broadening and deepening processes. All of them have been used with the purpose of consolidating a free, safe and prosperous environment for its citizens. With this objective in mind, the European Union has developed a particular vision on current relevant subjects in the fields of international relations in globalization, environment, democracy and global governance, which mark its international agenda, and in some cases, defining it as a cosmopolitan actor.

Based on this approach, the objective of this work is to make a brief description of the development of the European model of globalization from the beginning, and also to outline relevant trends in the near future. The description will come up with a political analysis that identifies the evolution of the constitutive principles of the European Union. The trends will be analyzed in two different ways, first considering the EU as a cosmopolitan actor, and second, considering the implications of the first analysis in its relations with Latin America, and with Colombia in particular.

\section{KEY WORDS}

The European Union, Latin America, Colombia, interdependence, trends, globalization, security, democracy, the cosmopolitan trend. 
del mundo. El desafío está en aprovechar esta preocupación para perfilar el futuro del modelo europeo y su papel en el exterior, dando respuesta, al mismo tiempo, a las tendencias integradoras que están marcando el orden internacional. En resumen, el objetivo es identificar un proyecto institucional global (Fontela, 2006).

Identificar este proyecto es obligación de las instituciones europeas; lo que se pretende aquí, es dilucidar cuáles son los caminos posibles. El propósito de este trabajo es, en primer lugar, realizar un breve balance de la conformación de la UE para entender en qué medida ha podido acercarse a un modelo cosmopolita y cómo esta conformación puede determinar su futuro. En segundo lugar, se pretende identificar el entorno al que se enfrentará a mediano plazo con la intención de conocer de qué manera puede cambiar su conformación y su papel en el sistema internacional, contribuyendo a hacerla más cosmopolita. Parte del análisis crítico en ambos apartados provendrán de las concepciones de Beck (2006) y Rosamond (2005) sobre la UE.

Finalmente, se considerarán los efectos de este contexto en las relaciones entre la UE y América Latina. Esta labor se realizará buscando las tendencias positivas hacia la interdependencia de dos importantes estudios prospectivos publicados recientemente por el Instituto de Estudios de Seguridad, pero siempre dejando entrever los efectos que puede tener un giro en ellas (Gnesotto y Ortega).

\section{El CAMino hacia la Unión EURopea}

\begin{abstract}
No cabe duda que la conformación de la Comunidad Económica Europea hace cincuenta años, no fue el resultado de la ejecución de un proyecto completamente consciente $y$ claramente definido. La firma del Tratado de Roma en 1957, fue el producto de una década construyendo consensos, así como de la convergencia de un conjunto de circunstancias que llevaron a seis países a concluir que la mejor opción para tratar algunos asuntos comunes, sería embarcarse en un proyecto novedoso como este (Parsons, 2002). En esa fecha no existía una idea clara y firme sobre lo que debía ser esa asociación. Sólo se contaba con la convicción de lo que no quería seguir siendo (un permanente foco de guerra) y la seguridad que la mejor fórmula para superar esto, era el consenso.
\end{abstract}

Desde el final de la Segunda Guerra Mundial y hasta 1957 el temor de incrementarse la tirantez entre Francia y Alemania y que esto desembocara en una Tercera Guerra Mundial, era constante. Durante esa década, como explica Parsons, se barajaron diversas fórmulas para evitar un terrible desenlace. Estos planes transcurrían desde el manejo diplomático usual hasta la conformación de una confederación de Estados (2002). En resumen, la tensión ante la amenaza no era diferente a ocasiones anteriores,;sin embargo, la solución sí lo fue.

Este desenlace positivo invita a escudriñar en los elementos que impulsan el proceso europeo de integración, ya que marcan las tendencias que han llevado a la UE al punto actual y que le encaminarán en el futuro. 
Desde esta perspectiva, el propósito de este apartado no es describir el proceso de integración europeo, suficientemente documentado en la actualidad, sino detectar algunos de esos elementos constitutivos que han estado presentes desde sus inicios y que aparentemente permanecerán en el futuro.

En las próximas líneas se describirán cuatro de esos elementos constitutivos y se comentará cómo determinan el proyecto europeo. En primer lugar, descubriremos brevemente la importancia del carácter progresivo del modelo, que tras cincuenta años le permite hacer un balance general positivo. En segundo lugar, revisaremos cómo el carácter normativo determina la forma de hacer las cosas en la UE y cómo esto potencia en algunos casos y, en otros, obstaculiza su desarrollo. En tercer lugar, se observará cómo ha avanzado en estos cincuenta años la concepción de seguridad de la Unión Europea, tan importante en sus inicios. Finalmente, se considerará cómo algunos principios fundamentales han determinado el estilo de convivencia europeo y descubriremos los retos a los que se enfrentan para seguir determinándolo en el futuro.

\subsection{Progresivo}

La intensa actividad entre Jean Monnet y Robert Schuman, apoyados en Alemania principalmente por Konrad Adenauer, a favor de crear unas condiciones que transmitieran tranquilidad y aseguraran un diálogo próspero entre las partes, y de generar progresivamente soluciones en los temas más delicados, no tiene precedente en la historia. Cuando a Francia comenzó a preocuparle la capacidad siderúrgica que estaba desarrollando Alemania en el Ruhr, se creó la CECA ${ }^{2}$ (1951); cuando a Europa le preocuparon las consecuencias del estallido de la guerra de Vietnam se intentó construir la frustrada Comunidad Europea de Defensa $(1952)^{3}$; cuando el desarrollo de la energía atómica o la competencia económica inquietó a estos países, se firmó el EURATOM y el Tratado de Roma en 1957 y así ha sido, como muestra el cuadro expuesto a continuación, desde entonces.

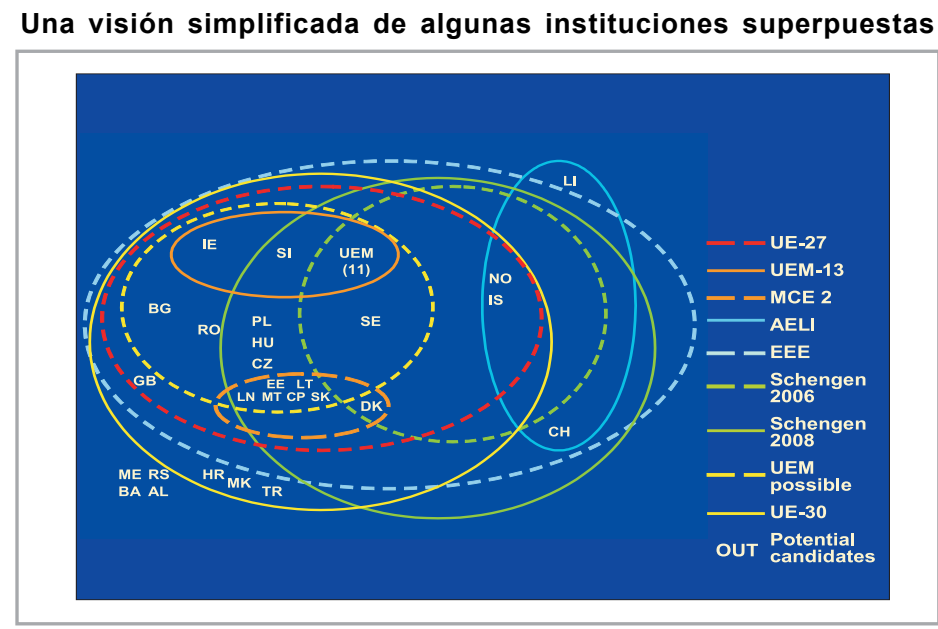

Fuente: Cavalier y Meunier (2006)

2 Comunidad Económica del Carbón y el Acero

3 Con el estallido de la guerra de Vietnam, debido a las tensiones este-oeste que se generaban, a Europa le preocupaban las consecuencias para Alemania dividida. También le preocupaba la posible salida de Estados Unidos del continente en caso que necesitara sus fuerzas en Asia, y la necesidad de permitir a Alemania comenzar la organización de sus fuerzas armadas. Este último aspecto es el que llevó a idear un aparato militar europeo que mantuviese controladas las capacidades e intenciones de cada país. 
Desde este punto de vista, la clave del éxito está en ir tratando los temas que más preocupan en común uno a uno, sometiéndose a intensos procedimientos de negociación que generan el consenso mínimo necesario para avanzar: "Evidentemente, hay que ir por etapas, a partir de sectores psicológicamente maduros, en los que facilidades técnicas específicas dejen prever un resultado espectacular..." (Schuman (1963), Pour L'Europe, Nagel, París, en Barea:80)

Esto marca el modelo europeo en dos sentidos: por un lado, es un proyecto a largo plazo y por otro, es un proyecto dinámico; es decir, cambia constantemente de forma tanto desde el punto de vista organizacional, como desde el punto de vista espacial. Ambos conceptos se conocen en la jerga de la UE como "la Europa de geometría variable" y "la Europa de dos velocidades" ${ }^{4}$. Son estas las características que nos permiten describir la Unión Europea en términos abiertos y hablar de un modelo en proceso, progresivo.

\subsection{Agenda normativa}

Explicado de un modo muy simple, el cosmopolitismo se fundamenta en cómo queremos que sean las cosas cuando se hacen correctamente. En el cosmopolitismo la acción viene guiada por el deber, es normativo.

Desde sus inicios, la construcción europea aspiró a estar marcada por el imperativo del deber. Como reconocen Jean Monnet y Konrard Adenauer en su momento, la propuesta de la CECA y la idea de seguir elaborando proyectos de consenso, no atendieron en su momento solamente al cálculo particular del interés nacional sino que en buena medida fueron también producto del deber ${ }^{5}$.

Para cumplir con este deber la UE se ha enfrentado a la dificultad de hacer ejecutivo lo normativo. Dicho de otro modo, aparte de la política económica y monetaria, el proyecto europeo se ha concentrado en desarrollar una intensa labor reguladora y en contar con un acervo comunitario que podría llenar más de una biblioteca. Este carácter normativo es beneficioso en la medida que le ha permitido entrar en muchos temas, que de haber deseado hacerlo desde el principio, utilizando solamente la fuerza ejecutora, se habría topado con grandes dificultades y fracasos seguros.

Bajo esta concepción, las instituciones europeas han sido capaces de coordinar la acción de temas relevantes para el conjunto europeo, pero le ha resultado difícil utilizar de manera determinante el poder que le permita llevar a cabo el ideal Europeo. La dificultad parece radicar en las herramientas utilizadas para desarrollar su labor. Como destaca Rosamond, especialmente en las relaciones externas de la UE, esta es marcadamente discursiva, aspiracional y declarativa (2005); de este modo parece que procura nombrar y conceptualizar temas, pero en algunos casos le resulta complicado darles forma y establecer agendas eficaces.

Es necesario reconocer que en conjunto el carácter normativo de la Unión es un valor. La importancia del deber que en la UE se materializa mediante lo que en inglés se conoce como "the rule of law", es una de las contribuciones de este modelo al orden mundial, como bien apunta Laïdi (2007).

\footnotetext{
4 Aunque a veces puede hablarse de "diversas velocidades".

5 “... Es la responsabilidad moral que tenemos para con nuestros pueblos y, no la responsabilidad técnica, la que debemos ejercer para realizar esta gran esperanza...” (Adenauer a Monnet el 23 de mayo de 1950, en Barea:50).
} 


\subsection{Espacio de seguridad}

Es también la UE un proyecto cosmopolita en la medida que desde sus inicios los esfuerzos se concentraron en eliminar la amenaza constante de guerra, dando paso al establecimiento de la concepción auténtica de $p a z^{6}$. En la actualidad, para los europeos es imposible pensar en algún problema que surja dentro de la región, que pueda originar una guerra entre sus miembros; ni siquiera que genere una tensión o amenaza previa a la guerra, que no pueda ser resuelto antes por otros medios.
El eurobarómetro, por ejemplo, muestra que la mayor parte de las preocupaciones de los europeos en este momento son asuntos de carácter nacional. Sólo el terrorismo, un problema que se percibe desde el exterior de la UE, es un elemento que genera sentimiento de amenaza conjunta y, que por el momento, pese a las diferencias en algunos aspectos de la política exterior, no parece ser capaz de fracturar la confianza de los ciudadanos europeos en su modelo de integración.

Asuntos que preocupan a los Europeos (EU 25)

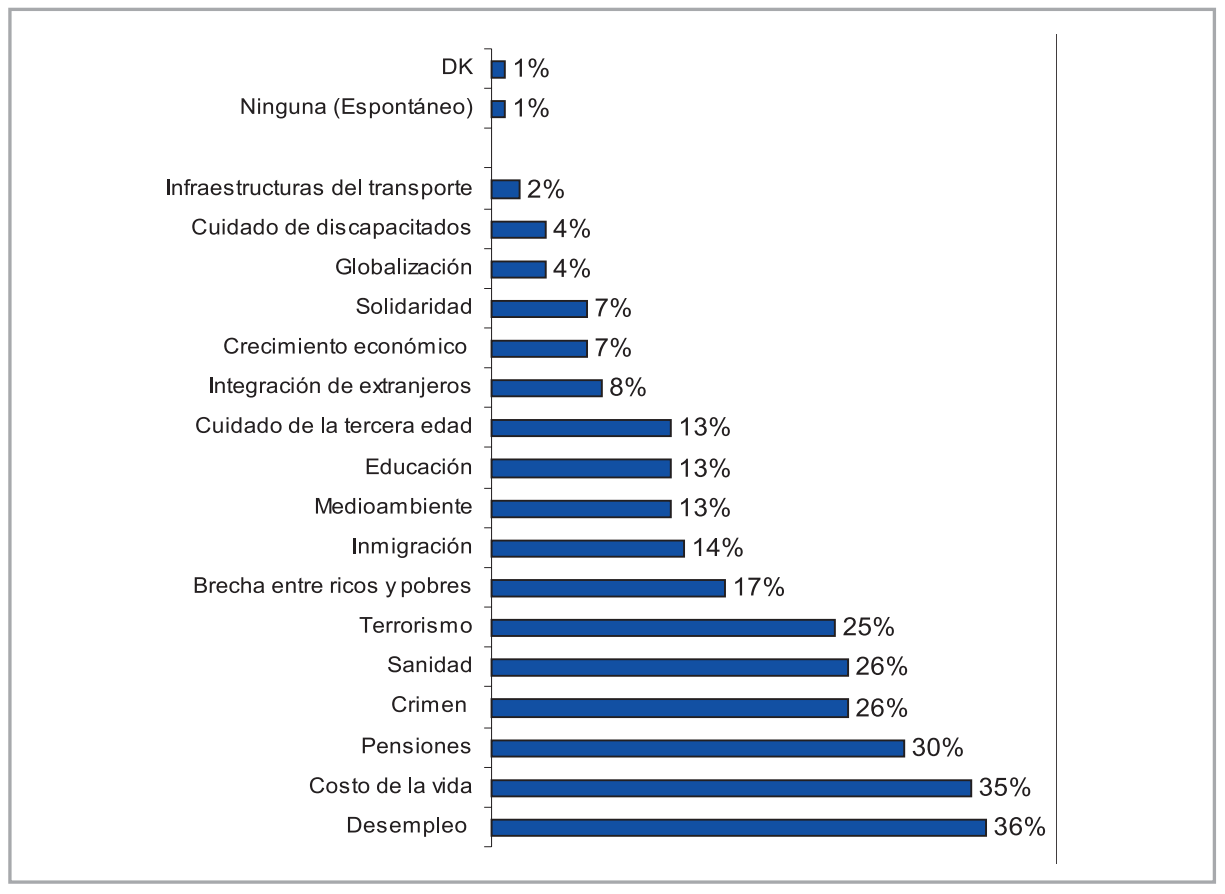

Fuente: Eurobarómetro 2006, p 43.

Esta observación deja entrever algunos restos del modo clásico de entender el sistema internacional, las amenazas y el equilibrio de poder, y es que la seguridad en la UE sigue considerándose en buena medida desde este punto de vista. Pese a haberse enfrentado a un terrorismo cuya conformación no está claramente definida y no guarda relación con

6 En relaciones internacionales existen tres fases de orden. El orden anárquico consta de la fase de guerra y de la fase de ausencia de guerra. No se considera que en ausencia de guerra exista paz, ya que no se eliminan completamente las tensiones ante la amenaza y en cualquier momento puede estallar la guerra nuevamente; el orden pacífico, el tercero, consiste en la ausencia prolongada de amenaza y, por ello, no existe posibilidad previsible de que brote la desconfianza y posteriormente la guerra. 
ningún Estado y enfrentarse a los desafíos del cambio climático (otro problema que no atiende a un formato estatal), a la Unión Europea le está costando crear soluciones no estatales a problemas no estatales. Esto puede llegar a percibirse como una decepción para la propia Europa y para algunos sectores de la comunidad internacional: que una figura que se ha caracterizado por encontrar soluciones creativas a envites complicados, no sea capaz de responder de la misma forma a las nuevas dificultades que surgen en el escenario internacional.

\subsection{Bienestar}

Desde un principio los arquitectos de la Unión Europea asumieron que no era suficiente la voluntad política y el anclaje institucional para que el proyecto europeo siguiera adelante; consideraron que estos eran dos factores que debían enmarcar los principios fundamentales que guían la acción dentro de la Unión Europea. Desde entonces son fundamentalmente cuatro los principios que se identifican constantemente en el discurso de la Unión: libertad, igualdad, solidaridad y diversidad.

Los dos primeros principios sellaron de un modo claro la relación de los seis países que firmaron el Tratado de Roma. Pese al desenlace de la Segunda Guerra Mundial y la creciente tirantez entre Francia y Alemania en el periodo de reconstrucción, el proyecto se concibió para integrar a alemanes y franceses en el plano de la igualdad y la libertad ${ }^{7}$.
Desde el punto de vista del acceso puede afirmarse que, en términos generales, de partida, todos los ciudadanos y países cuentan con igual derecho de acceso y garantía a sus libertades, teniendo en cuenta, por supuesto, el debido proceso de adaptación, que en ocasiones restringe libertades no fundamentales como la de movilidad dentro de todo el espacio europeo. De forma generalizada, la UE cuenta con países democráticos y con libertad económica, lo que en principio le erige como un espacio de libertad consolidada.

Una vez dentro, el modelo social europeo no ha logrado completar el principio de igualdad a lo largo de sus cincuenta años de existencia. Actualmente, el principio de igualdad material ${ }^{8}$ se cumple en la UE mediante el sistema de bienestar, establecido nacionalmente con escasa coordinación con las instituciones supranacionales. Es decir, el cumplimiento del principio de igualdad depende de la concepción de Estado de Bienestar adoptado por cada Estado miembro, generando, por lo tanto, diferencias entre países.

Relacionado con el principio de igualdad está el principio de solidaridad ${ }^{9}$. Si bien es cierto que para todos los ciudadanos de la UE aún no se ha podido establecer una política de bienestar que fomente la igualdad, bajo el principio de solidaridad, sí se intenta proporcionar esa igualdad a las regiones menos desarrolladas.

\footnotetext{
${ }^{7}$ Es decir, todos los miembros entraron en el proceso en igualdad de condiciones sin ser juzgado su pasado y, por ello, condenar su futuro dentro del nuevo orden.

${ }^{8}$ Beck (2006) establece claramente la diferencia entre igualdad material e igualdad no material; esta última relacionada con la aceptación de la diversidad cultural y racial que afecta la relación de igualdad desde el punto de vista de la diversidad como avanzaremos brevemente más adelante.

${ }^{9}$ Igualmente, este es un principio propuesto en los inicios del proyecto europeo: "Todas estas tendencias [nacionalistas segregadoras consecuencia de la guerra] que nos ha legado el pasado habrá que sustituirlas por la noción de solidaridad; es decir, la convicción de que el verdadero interés consiste en reconocer y aceptar en la práctica la interdependencia de todos" (Schuman, Barea: 80)
} 
Actualmente, son los Fondos Estructurales, como parte de la política regional, los representantes por excelencia de la materialización de este principio. No obstante, la realización del principio de solidaridad basado en el criterio territorial de la política regional debilita la relación causal solidaridad-igualdad y trae a debate la cuestión sobre el territorio, como la unidad en la que se basa el desarrollo espacial y organizacional de la Unión ${ }^{10}$. Dicho de otro modo, fundamentar la transferencia de recursos hacia los grupos de riesgo en criterios territoriales, diluye la eficacia de la acción solidaria, enfocada a lograr igualdad entre todos los ciudadanos de la UE.

Desigualdad en la distribución del ingreso

(ratio de ingreso por quintil)

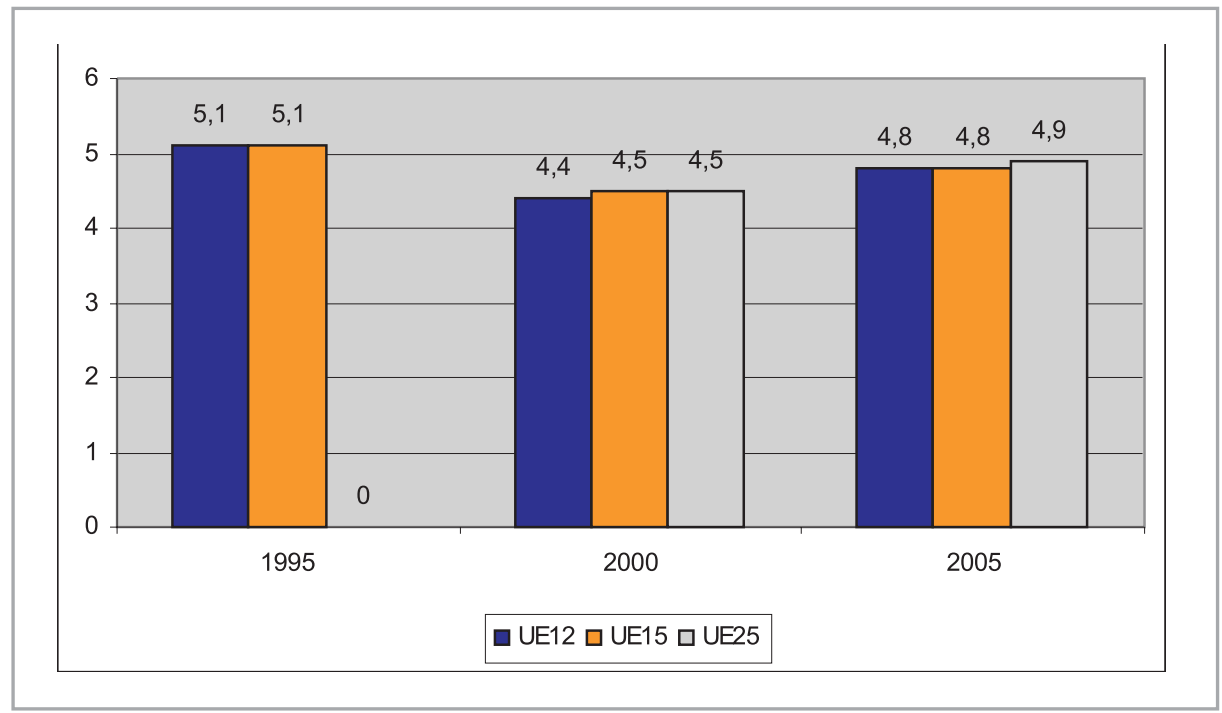

Fuente: Eurostat 2006

La consideración de la Unión Europea como un aparato espacial y temporalmente indefinido, compuesto por una variopinta? relación de naciones que a su vez cuentan con múltiples culturas, sociedades y sistemas, permite definirla como un espacio de diversidad. Esta diversidad se afianza en la concepción de ciudadanía.

La ciudadanía europea no elimina la ciudadanía nacional; por el contrario, la complementa. En este sentido, la ciudadanía europea se convierte en un paraguas que acoge grupos nacionales claramente diferenciados. No obstante, el incremento de esa diversidad se ha alcanzado como consecuencia de la ampliación de fronteras. Como explica Beck, la diversidad europea no atiende a un criterio dinámico y de movilidad (2006). Es decir, los europeos cuando pensamos en diversidad, pensamos en los franceses dentro de su país, en los irlandeses dentro de su país o en los húngaros dentro de su país; no pensamos en los italianos repartidos por todo el territorio

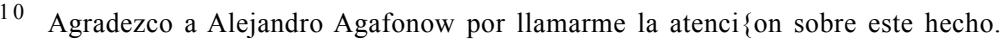


europeo y mucho menos en los rusos o estadounidenses, en parte debido a la escasa movilidad de los ciudadanos entre países miembros. Esto afecta con frecuencia la concepción real de la diversidad, que no permite ver, por ejemplo, el aporte que a esta pueden hacer minorías destacadas, como los 17,6 millones de extranjeros que habitaban en la Unión en 2005 (Eurostat) ${ }^{11}$. Es así como se ve afectada la relación causal diversidad-igualdad.

Este panorama muestra que, aparte del reto diario que estos principios imponen en la actualidad a las instituciones europeas, a largo plazo se enfrentarán a una prueba crucial. Una vez que se encuentren plenamente integrados los países de Europa del Este, gracias a la caída del Muro de Berlín que consideró su entrada a la UE como algo casi natural, cuando no un deber, estos valores pasarán una prueba decisiva en futuras ampliaciones, posiblemente menos asimiladas.

En retrospectiva, no cabe duda que en el transcurso de cincuenta años estos principios han sido cruciales en el avance del proyecto y que son muchos los logros alcanzados. Pero tampoco cabe duda que en el futuro, para seguir esta tendencia, será necesario volver constantemente a ellos y determinar el contenido que deseamos darle.

\section{3. ¿Puede ser la Unión Europea más cosmopolita?}

Aunque existen elementos que pueden desviar el curso actual del orden internacional, las principales tendencias generales en las que la UE estará sumergida en los años próximos pueden puntualizarse del siguiente modo (Ortega, 2007):

- Protección y fomento de los valores comunes del sistema internacional. ${ }^{12}$

- Creciente importancia del calentamiento global.

- Creciente importancia del terrorismo internacional.

- La economía mundial girará en torno a los países emergentes y sus relaciones con los países desarrollados.
En consonancia con estas previsiones, la Unión Europea, en su celebración del 50 aniversario, dispone, como cuatro, los retos a los que tendrá que enfrentarse en el futuro: la globalización, el medioambiente, su papel en el mundo y el fomento de la democracia. Son estos elementos los que perfilarán el modelo europeo en el futuro y los que marcarán los contenidos de los elementos constitutivos antes comentados.

En las próximas líneas se verá cómo las tendencias de los cuatro retos mencionados anteriormente, marcarán la agenda de la UE, tanto interna como internacionalmente, en la consolidación de sus principios constitutivos de seguridad y bienestar.

11 Esta es una cifra que supera la población total que habita 18 países miembro de la Unión.

12 Ortega explica que los principios que en la actualidad la comunidad internacional protege y promueve son, fundamentalmente: mantenimiento de la paz, democracia, libre comercio, protección del medioambiente y multilateralismo, a los que se unen otros que se han asimilado a lo largo del siglo XX como por ejemplo, los principios consagrados en la Carta de las Naciones Unidas (Ortega, 2007:30-32). 


\subsection{La democracia}

En la UE la democracia es al mismo tiempo un valor asimilado y un instrumento por establecer. Es un valor asimilado en la medida que cada Estado miembro debe basar su organización política en un sistema democrático; también lo es en tanto constituye una cláusula insalvable en todo acuerdo celebrado por los países miembros o por la UE con terceros. Pero igualmente es un instrumento por establecer en cuanto las instituciones europeas aún adolecen del déficit democrático, numerosas veces nombrado en diversos análisis sobre el modelo europeo.

Como se analizó en la primera parte de este trabajo, el avance a medio plazo del modelo europeo se basa en establecer instrumentos democráticos más eficaces. Al respecto, Ortega puntualiza entre los factores internos determinantes para el futuro de la Unión asuntos como, por ejemplo, las relaciones entre gobiernos y su capacidad para trazar visiones comunes, 0 el proceso de toma de decisiones (2007).
Las instituciones europeas son conscientes de la importancia de transformar los procedimientos de toma de decisiones y de la necesidad de acercar este poder a los ciudadanos.

El Tratado Constitucional es un reflejo de la dificultad que impone materializar esta transformación. Tras el fracaso en su ratificación, en la reflexión posterior para identificar los puntos débiles del proceso, los propios representantes europeos apuntaron a la falta de inclusión de los ciudadanos europeos en el proceso de elaboración. Es curioso, que después de llegar a esta conclusión, el pasado 23 de junio en la Conferencia Intergubernamental de Bruselas se haya adoptado una Constitución en la que de ningún modo participaron, ni está previsto que participen, los ciudadanos europeos. Está claro que la UE no podía permitirse otro fracaso en este asunto, pero es probable que los dirigentes europeos no hayan considerado que la clave del éxito no radica solamente en la aceptación del documento y de tratar de dar una forma más clara a la UE, sino en la asimilación de las reglas democráticas.

\section{Fomento de la democracia y la participación}

Ante los retos de los próximos 50 años, la Unión Europea necesitará unos instrumentos jurídicos y de gestión mejores, que no tiene todavía. El primer intento en este sentido fracasó cuando, en 2005, los votantes de Francia y los Países Bajos rechazaron el proyecto de Constitución de la UE. Los mandatarios de la Unión reconocieron que ello se debió en parte a que los ciudadanos se sienten mal informados sobre Europa y excluidos de sus decisiones y, ante esta situación, se pusieron en marcha varias iniciativas para retomar el diálogo con ellos y darles más voz en los futuros asuntos políticos, entre los cuales están las reformas institucionales necesarias en los próximos años para garantizar el funcionamiento eficaz de la Unión en el siglo XXI.

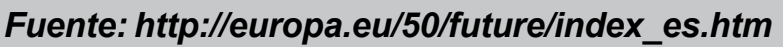


Desde el punto de vista internacional, la UE constituye un importante promotor de la democracia; sin embargo, la mayor parte de su actividad en este ámbito se concentra en las relaciones entre Estados y se reduce a la exigencia del cumplimiento de principios formales de la democracia, en vez de dedicarse al fortalecimiento de los valores y confianza en sistemas de este tipo.

La democracia debe promoverse también entre las instituciones internacionales ya que esto fortalecerá el papel europeo y mejorará su eficacia. No cabe duda entonces, que esta labor debe realizarse también conjuntamente con organismos como las Naciones Unidas, a la que debe aportar su experiencia y apoyo para transformarla en una institución eficiente que responda a las necesidades actuales de la comunidad internacional.

\subsection{Seguridad}

En la Estrategia Europea de Seguridad presentada en 2003 por el Consejo, se perfila el problema de la seguridad como un tema que no concierne exclusivamente al aparato militar (CUE, 2003). En este documento se concibe la seguridad europea como un asunto que depende tanto de la estabilidad en su entorno, como de su propia acción para fomentarla. El aporte de la Unión Europea al orden internacional radica en su insistencia respecto a que la estabilidad no depende del equilibrio de poder en términos militares, sino de cuestiones como el desarrollo, el comercio o el medioambiente. Por esta razón, en las próximas líneas se estudiarán las tendencias medioambientales y de gobierno global que marcarán el comportamiento de la Unión a mediano plazo.

\subsubsection{Medioambiente}

Desde el punto de vista medioambiental, la UE ha procurado ser proactiva en las iniciativas internacionales para atacar el problema del calentamiento del planeta, cumpliendo de manera global, por ejemplo, con las emisiones de gases de efecto invernadero establecidas por el Protocolo de Kyoto. Según el Eurobarómetro, el cambio climático es uno de los temas que preocupa a sus ciudadanos (el $50 \%$ mucho y el $37 \%$ hasta cierto punto); la mayoría considera que este debe tenerse en cuenta en toda Europa (62\%). Los ciudadanos europeos son conscientes de los cambios que sufrirán a corto plazo en su estilo de vida, debido a las medidas que se deben tomar para atacar este problema (Eurobarómetro, 2007).Con base en esto y en el acceso de la región a fuentes energéticas, la UE ha desarrollado una política notablemente activa, aunque no fácil para superar las dificultades de acceso y lidiar con el dilema energía-crecimiento.

Por un lado, la política energética europea se está concentrando en diversificar sus redes de abastecimiento de gas y petróleo, apoyando la construcción de oleoductos y gaseoductos en el Mediterráneo, así como estrechando sus relaciones con Rusia. Este país es el mayor socio proveedor de petróleo y gas a Europa y asegurar una relación estable y segura con él significa un intenso trabajo, especialmente desde la intensificación de las relaciones Sinorusas tanto bilaterales como multilaterales ${ }^{13}$.

Esto obliga igualmente a las instituciones europeas a concentrarse poderosamente en la diversificación e intensificación del uso de fuentes renovables para superar las dificultades del acceso a fuentes energéticas tradicionales.

13 En estas relaciones el tema energético es uno de los primeros puntos en la agenda y China se está convirtiendo cada vez más en un socio importante para tirar de la economía rusa (Fontela y Pérez, 2007). 
Por otro lado, del dilema energía-crecimiento cabe resaltar, por ejemplo, que en el ámbito público España es uno de los dos países (junto con Chipre) más preocupados por el cambio climático (Eurobarómetro, 2007). En este sentido, parece coherente que sea uno de los países que más intensamente está apoyándose en la producción de energías renovables y, por ende, que esté a la cabeza de la mayor parte de las energías de este tipo, consideradas en la política energética europea (Observ'ER, 2006). No obstante, refleja la dificultad de enfrentar este tema, que también sea España el segundo país (tras Turquía) que más incumple con las cuotas establecidas de emisión de gases de efecto invernadero de todos los signatarios del protocolo de Kyoto (+49\% en 2004 según la UNFCCC).

No cabe duda que para el crecimiento y bienestar de la UE, considerar el problema del cambio climático como un asunto de seguridad es fundamental para poner la atención necesaria en su solución. La tendencia global por considerar este asunto como tema prioritario en la agenda y el carácter normativo llevará al proyecto europeo a reforzar esta visión y a buscar un modelo económico más viable en términos medioambientales.

\subsubsection{Gobierno global, desarrollo y terrorismo}

El papel de la UE como actor singular y relevante en el escenario internacional es otro punto por tener en cuenta en el apartado de la seguridad. Disfrutar de un entorno estable y próspero es fundamental para la evolución positiva de Europa. No cabe duda que el tema medioambiental podría ser uno de los que determine esta estabilidad y prosperidad en ámbitos específicos (en caso de guerras por acceso a recursos naturales), pero considerar otras tendencias generales que determinan el orden global permitirá prever la contribución que la UE puede hacer y cómo, a su vez, esta evolucionará internamente.

En los pocos años transcurridos del siglo XXI, los valores de la sociedad internacional se han transformado notablemente y están conformando un futuro diferente. Como explica Ortega, de continuar predominando el criterio del balance de poder y el "auge y caída de las grandes potencias", propio de la Guerra Fría, y observando su auge económico actual, los BRIC ${ }^{14}$, Japón y la Unión Europea deberían estar construyendo alianzas estratégicas y rearmándose al más puro estilo "realpolitik" (2007). En contraposición, en las últimas décadas se ha perdido el interés en medir el poder en el escenario internacional, en relación con la capacidad militar.

El ritmo económico, el nivel de innovación, el capital humano y social, la actitud política o la interdependencia son algunos de los factores que hoy en día condicionan el poder y su diferente intensidad y combinación en cada país y región.

De este modo, el régimen internacional se ha transformado, perdiendo influencia el balance de poder clásico y prevaleciendo la multipolaridad. Actualmente, la estrategia internacional, incluso del país más pequeño, observará cuánto hace o deja de hacer Estados Unidos, pero no podrá obviar sus intereses comerciales, económicos, políticos o energéticos relacionados con la UE, Japón o los BRIC. Así, se está estructurando un orden internacional multipolar en el que "los poderes relevantes no son homólogos" (Fontela y Pérez, 2007).

14 Acrónimo utilizado para nombrar los países emergentes: Brasil, Rusia, China y la India. 
Además de la diversidad de poderes estatales relevantes en el sistema internacional, cada vez toman más importancia actores no estatales (cuyo auge se produjo hace un par de décadas) miembros plenos, hoy en día, del sistema internacional. ONG, foros de discusión internacional, organismos multilaterales y otras formas de movilización de la sociedad civil están logrando posicionar sus demandas y perspectivas entre los primeros puntos de la agenda del sistema internacional,y son en la actualidad interlocutores de pleno derecho ${ }^{15}$.

El regionalismo es otra tendencia que, aunque no es novedosa y de la que no se prevén grandes transformaciones en los acuerdos existentes actualmente, si es posible que su evolución y consolidación sea cada vez mayor, especialmente en torno a los países emergentes. Procesos de integración como el MERCOSUR en América Latina o la SCO en Asia tienen posibilidades de adquirir mayor peso en la escena internacional.

En resumen, multipolaridad, actores de diversa composición y la integración son tres aspectos que la UE concibe dentro de su planteamiento de gobierno global ${ }^{16}$. Un orden internacional que refuerce estas tendencias permitirá a la UE consolidar un espacio de seguridad en los términos que se han propuesto al principio de este documento y permitirá construir los puentes necesarios para extender a otros países su concepción sobre un sistema internacional estable y próspero.
Desde el punto de vista geopolítico, son dos las tendencias que desde el final de la Guerra Fría están marcando el orden internacional. Como explica Ortega, por un lado, el rechazo a la guerra como mecanismo para la resolución de conflictos tanto por parte de países democráticos como de países no-democráticos; por otro lado, la tendencia a buscar temas comunes para establecer relaciones pacíficas (lo que ha incrementado la variedad de actores en la escena internacional) ${ }^{17}$. Pero si las grandes tendencias apuntan a un escenario general pacífico, debe reconocerse que la guerra sigue siendo un elemento latente, siempre dispuesto a estallar cuando la voluntad política y las estrategias de desarrollo fallan.

Tras el primer ataque terrorista internacional, el 11-S, eran frecuentes los análisis que consideraban como una de las principales causas de este terrorismo, la incapacidad del sistema internacional y, especialmente de los países que lo lideran, para aportar soluciones que generaran un entorno económicamente próspero y social y políticamente estable. Esta perspectiva ha permanecido junto a una muy reforzada política de seguridad. Desde este punto de vista, las políticas de desarrollo siguen marcando la agenda de las relaciones internacionales; sin embargo en el futuro más atención se podrá dedicar al tema del desarrollo a medida que la amenaza terrorista se materialice con menos frecuencia.

15 En este ámbito, el problema del calentamiento global es un ejemplo de movilizaci \{on que ha obligado a cambiar las prioridades de muchos gobiernos.

16 Entendiendo este último término como mecanismos de regulación de las relaciones en el sistema internacional. En inglés se puede hacer una distinción muy clara sobre este tema. Como explica Ortega, existe una diferencia clave entre "global governance" y "global government". En el primero, el orden internacional se rige por una red multiforme de instituciones tal como observamos hoy en día; mientras, que en el segundo debe existir una estructura supranacional claramente definida que regule todas los aspectos de las relaciones internacionales. Desde este punto de vista, la palabra que definiría mejor esta relación sería "gobernanza global".

17 Esta es la tendencia general, ya que de modo particular sigue siendo Medio Oriente el principal foco de conflicto internacional (Ortega, 2007). 
No cabe duda que la agenda de desarrollo favorece más al proyecto y a los objetivos de la UE, aunque, como de costumbre, habría que poner la lupa sobre la ejecución y los verdaderos resultados de las políticas de desarrollo. Está claro que normativamente la UE concibe este asunto como parte de la solución a muchos problemas en el mundo (como el terrorismo), y como un asunto que debe atenderse desde diversos enfoques, como muestran los ámbitos de acción de la política de desarrollo, ejecutados por la Dirección General de la Comisión especializada en este tema.

\section{Políticas de Desarrollo}

Areas de intervención: Integración Regional y Comercio Prevención del conflicto Democracia y derechos humanos

Asuntos transversales: Igualdad de género Democracia y derechos humanos

Fuente: http://ec.europa.eu/development/PoliciesGen_en.cfm

\section{Medioambiente Infraestructura \\ Desarrollo rural Desarrollo humano \\ Dimensión social Agua y Energía}

VIH/SIDA Medioambiente
En caso de poder desplegar esta agenda en un contexto medianamente propicio y de contar internacionalmente con el fortalecimiento de tendencias integradoras, la política de la UE en este ámbito deberá considerar cada vez más una agenda que se apoye en la perspectiva del codesarrollo, ya que en un mundo interdependiente queda en evidencia la ineficacia de políticas trazadas desde una sola perspectiva y ejecutadas con escasa participación de sus receptores (ver Naïr).

En resumen, para construir un espacio de seguridad, como lo concibe la UE, y teniendo en cuenta que es probable que las tendencias negativas (excepto las energéticas) no se agudicen, la UE deberá trabajar intensamente a favor del fortalecimiento de las tendencias positivas; esto no podrá hacerlo sin la ayuda de otros actores interesados igualmente en el predominio de este escenario como podría ser el caso de América Latina, los países emergentes o procesos como la SCO cuya historia reciente se ha visto penosamente marcada por la necesidad de combatir el terrorismo internacional en detrimento de otros objetivos (Fontela y Pérez, 2007). Por último, concebir las tendencias medioambientales y energéticas como un elemento de consenso en la comunidad internacional contribuirá a reforzar las tendencias positivas.

\subsection{Globalización}

Mantener el ritmo en un mundo globalizado significa poner a prueba la capacidad innovadora de la región, así como la intensificación de su integración económica y financiera. En la UE ambos aspectos se apoyan fuertemente en el futuro demográfico y en el nivel de bienestar, pues estos son elementos que pueden poner en tensión el avance o retroceso de Europa en la 
globalización, debido a que son el capital humano y el capital social los que en mayor medida aportan la creatividad y el dinamismo necesarios. La transición demográfica afectará la cantidad y calidad de la población activa necesaria para mantener la marcha económica que mantiene a Europa dentro de la globalización.

Hoy en día, es ampliamente conocido por todos los europeos que la región se está enfrentando a un continuo proceso de envejecimiento como consecuencia de las bajas tasas de natalidad y mortalidad, así como por el incremento de la esperanza de vida. También es conocida la presión migratoria que experimentan algunos países de la región.

Esta situación desafía a Europa de dos formas adelantadas en el primer apartado. Por una parte, exige de las instituciones europeas políticas más eficaces, propensas al mantenimiento del bienestar ciudadano. El problema del envejecimiento de Europa se entiende cada día más como un problema transnacional, como uno de esos problemas comunes que están esperando ser tratados por las instituciones europeas con determinación. No parece ser suficiente la declaración de directivas que definen temas y coordinan voluntades como ocurre con el Consejo de Lisboa de 2000 que propone constituir en 2010 la "más competitiva y dinámica economía del conocimiento en el mundo, basada en crecimiento económico sostenible, con más y mejores trabajos y mayor cohesión social", y cuyos objetivos se posponen año a año, frenando la capacidad de liderazgo europeo en la globalización.

Cumplimiento de los objetivos del Consejo de Lisboa

\begin{tabular}{|l|c|c|}
\hline & Objetivo & $\mathbf{2 0 0 5}$ \\
\hline Tasa global de empleo & $70 \%$ & $63,8 \%$ \\
\hline Empleo femenino & $57 \%$ & $55 \%$ \\
\hline Trabajadores de mayor edad & $50 \%$ & $42,5 \%$ \\
\hline
\end{tabular}

Fuente: Consejo Europeo, 2007

Por otro lado, esta situación genera una presión importante sobre el actual concepto estático de diversidad, que la empuja fuera de los argumentos nacionales y la obliga a introducirse en la consideración de problemas que afectan a las minorías en la región, igualmente de un modo transnacional, o mejor dicho, europeo. Como apunta Beck, la UE es una región de fronteras cada vez más débiles internamente y cada vez más reforzadas externamente. Este hecho unido a la definición nacional de las políticas migratorias que no permite ver a los Estados más allá de sus preocupaciones particulares sobre el tema, lleva a que los países del norte, por ejemplo, no entiendan y no se impliquen en las consecuencias de la presión migratoria que reciben en estos momentos los países mediterráneos como España e Italia; así como tampoco permite a estos últimos comprender las políticas migratorias de sus coterráneos del norte. El resultado final es una situación desfasada entre presión migratoria y la migración necesaria para que Europa mantenga los ritmos de crecimiento e innovación necesarios. 


\section{Tendencias demográficas a 2025}

- En 2025 la Unión Europea constituirá sólo el 6\% de la población mundial.

- Aunque el número de migrantes ha decrecido ligeramente, el número de migrantes internacionales que se dirigen hacia países desarrollados está en crecimiento.

- En 2005 Francia, Alemania, Italia, España y el Reino Unido recibieron más de 29 millones de inmigrantes internacionales.

- Se debe resaltar que desde finales de los años ochenta el crecimiento demográfico en la UE es más bien el resultado de la inmigración que del crecimiento natural.

Fuente: Gnesotto, 2005

Las tendencias demográficas, la necesidad de seguir el ritmo de crecimiento utilizando un modelo económico que evite mayores daños al medioambiente, así como la amenaza del terrorismo internacional, presionarán a esta región para que se preocupe más por lo que ocurre fuera de sus fronteras y para que adopte una visión tendiente al co-desarrollo. Es esta la vía para encontrar el equilibrio entre políticas migratorias coherentes con el crecimiento de Europa y con la estabilidad del entorno internacional necesario.

Este hecho confirma, además, que paulatinamente el fenómeno de la globalización repercutirá cada vez más en asuntos políticos, sociales y culturales.

Desde el punto de vista económico, a mediano plazo, las tendencias parecen mantenerse (Gnesotto, 2005):
- Primacía de las economías avanzadas (EEUU, UE y Japón).

- Deslocalización, especialmente hacia países emergentes.

- Crecimiento del comercio mundial y con especial fuerza de los movimientos de capital.

Estas tendencias reflejan claramente las consecuencias de la creciente interdependencia de los países avanzados y, especialmente de la UE, y nos permiten adivinar con facilidad cómo pueden determinar la acción de la región.

Pese al intenso crecimiento y desarrollo de los países emergentes, aún queda tiempo para que constituyan una verdadera competencia para la UE. Teniendo en cuenta que el crecimiento económico se está apoyando cada vez más en la innovación y, específicamente, en el progreso tecnológico ${ }^{18}$, el proceso europeo requiere trazar

18 La India es el primer país del mundo en servicios tecnológicos y la demanda de China en productos de este tipo es cada vez más intensa. Ver Fontela y Pérez.2007. 
una estrategia a largo plazo para no quedar rezagada. Este no es un análisis novedoso. Como se ha comentado anteriormente la Estrategia de Lisboa de 2000 tenía este propósito al promover el incremento del gasto en I+D al 3\% en 2010. No obstante, al integrar este reto a las tendencias energéticas y a las crecientes exigencias medioambientales, se presenta un serio dilema, sin contar, una vez más, con la necesidad de superar el inmovilismo que hay entre las fronteras nacionales europeas que frenan la consolidación de un espacio de educación óptimo y un verdadero mercado de trabajo europeo.

Intensidad de I+D (gasto en I+D como \% del PIB) por sector,

EU25 y países seleccionados - 2004

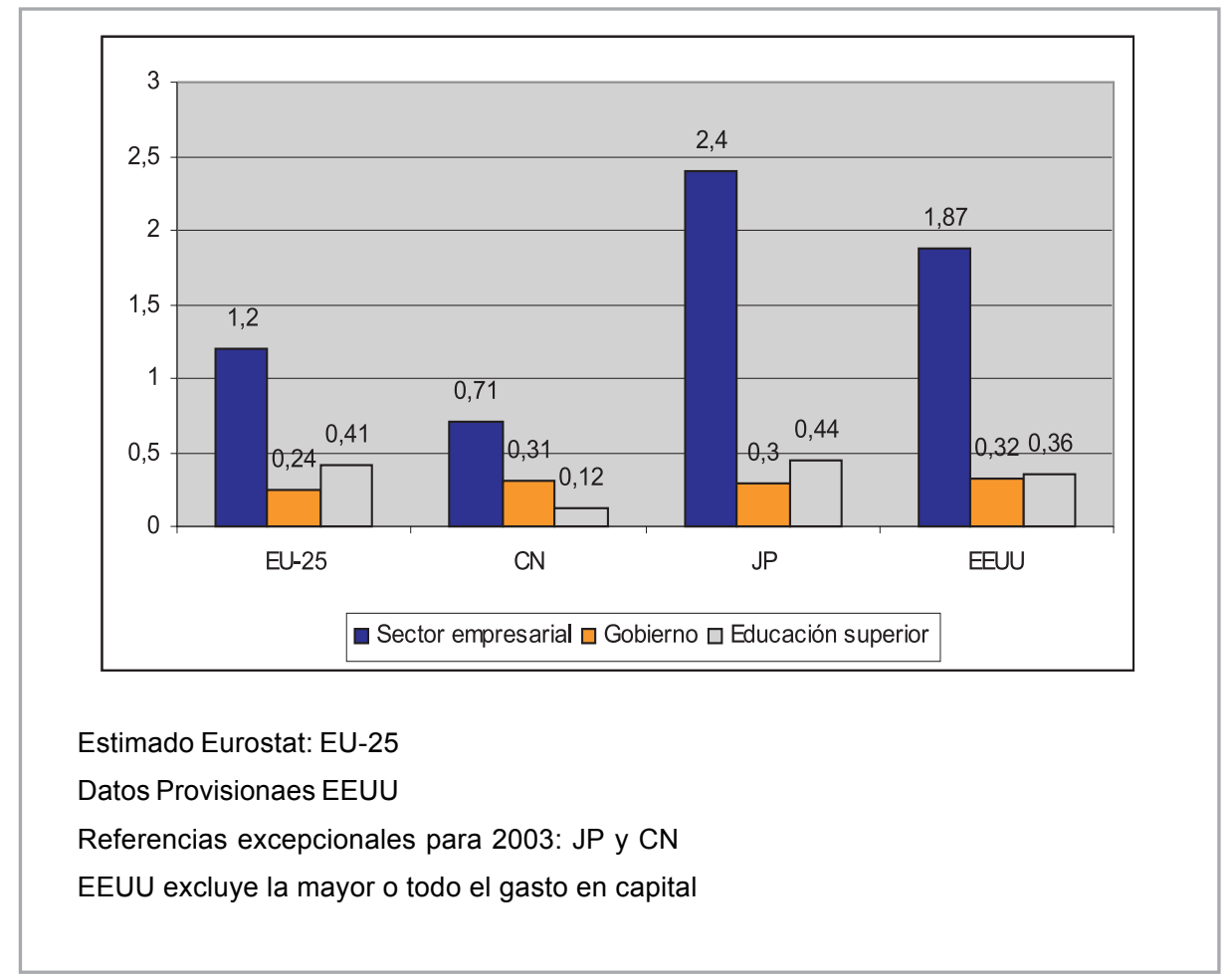

Fuente: Eurostat, 2007 


\section{LOS EFECTOS DEL COSMOPOLITISMO EUROPEO EN LAS RELACIONES CON AMÉRICA LATINA}

$\mathbf{E}_{1}$

recorrido que se ha hecho hasta estas líneas sobre la construcción y el devenir de Europa pretende ser útil para los que nos preocupamos por el futuro de América Latina y de Colombia en dos sentidos.

Por un lado, debe servir para reflexionar sobre los elementos comunes que pueden ayudar a las diversas regiones latinoamericanas a apoyarse sobre pilares sólidos, y así vigorizar sus procesos de integración y alcanzar los objetivos que se han propuesto.

Pese a la extendida percepción del proyecto europeo como un proceso de mera integración económica, se observa que en buena parte han sido los aspectos económicos los que han contribuido con la consecución de objetivos no económicos. Esto nos indica que las regiones latinoamericanas deben ir más allá de sus relaciones comerciales, identificar los asuntos que les unen, los que les separan y los que les preocupan por igual (el acceso al agua, el acceso a recursos energéticos, los grupos armados, la producción y el tráfico de droga, la conservación de la selva amazónica, las redes de transporte) y estrechar lazos, construyendo redes tema a tema, asimilando que los efectos no son automáticos, que el camino será largo y que habrá algún fracaso, pero que en conjunto se habrá entrado en una dinámica positiva ${ }^{19}$. No cabe duda que esto requiere una voluntad política que va más allá de lo cotidiano y lo electoral, lo cual dependerá del trabajo de gente, si no brillante, sí muy hábil para construir alianzas.

En segundo lugar y, más importante, debe darnos luces sobre las oportunidades que tiene América Latina para engancharse en la futura dinámica europea, llamar su atención y crear sinergias.

La estabilidad demográfica, la carencia de graves conflictos y un ritmo económico más o menos constantes (Gnesotto, 2005), hacen que los desafíos a los que se enfrentará América Latina a mediano plazo sean considerablemente diferentes al resto del mundo en cuanto a su contenido.

Desde el punto de vista de amenaza a la seguridad, América Latina está fuera de la agenda europea, como muestra, por ejemplo, la relación de operaciones de la UE en el mundo de la Estrategia de Seguridad. Este es un punto a favor de la región ya que la hace un socio estable y le permite concentrar la atención de ambas partes en temas constructivos, como por ejemplo, el económico.

19 No debe esperarse que en América Latina se pongan en marcha diversos procesos de integración que sigan uno a uno los pasos que ha dado la UE (Rosamond, 2005), lo que se sugiere es que la integración requiere identificar elementos comunes que a medida que se abordan con éxito llevan al siguiente paso y así, sucesivamente. 


\section{Operaciones en curso de la UE}

\section{Balcanes Occidentales}

- Operación militar en Bosnia y Herzegovina (EUFOR-Althea).

- Misión policial en Bosnia-Herzegovina (EUPM) .

- Equipo de planificación en Kosovo.

\section{Medio Oriente}

- Misión policial en los territorios palestinos (EUPOL COPPS).

- Misión de asistencia fronteriza en el puesto de Rafah en los territorios palestinos (EU BAM Rafah).

- Misión en Iraq para el cumplimiento de la ley (rule of law), (Eujust Lex).

\section{Asia}

- Misión Policial en Afganistan (EUPOLAFGHANISTAN).

Africa

- Misión policial en Kinshasa (DRC) (EUPOL Kinshasa).

- Misión para la reforma del sector seguridad en la república Democrática del Congo (EUSEC DR Congo).

- Apoyo a AMIS II (Darfur)

Fuente:http://www.consilium.europa.eu/cms3_fo/showPage.asp?id= 268\&lang= ES\&mode=g

Las posibilidades de América Latina y, Colombia específicamente, se concentran más en el lado de las tendencias positivas que de las negativas. Si utilizamos la relación de fuerzas negativas que ofrece Ortega, se observa que América Latina puede estar presente en muy pocas $^{20}$.

Sin embargo, América Latina sigue sin lograr ser un foco de atención importante para la comunidad internacional, debido tanto a las dificultades para transformar su crecimiento en desarrollo, como al despunte de las economías asiáticas que opacan sus medianos logros.
La buena fortuna en el ámbito de la seguridad y el escaso desempeño económico, obligan a este continente a buscar formas ingeniosas de llamar la atención de los líderes de la comunidad internacional.

Desde el punto de vista de la gobernabilidad, Colombia es una de las pocas democracias maduras en América Latina, aunque a mediano plazo los factores que constantemente ponen en juego su estabilidad, permanecerán. Colombia seguirá enfrentándose al desafío de aplacar los movimientos guerrilleros y como el resto de países latinoamericanos deberá enfocarse en

20 Estas fuerzas las resume en siete puntos:

1. Confrontación entre poderes globales.

2. Guerras en medio Oriente.

3. Cambio climático, guerras por recursos naturales.

4. Catástrofes humanitarias en África y pobreza extrema.

5. Rearme en particular con armas de destrucción masiva.

6. Crisis económicas y financieras globales.

7. Crimen Organizado y terrorismo. 
alcanzar el desarrollo, especialmente fomentando la igualdad. En caso de sortear estas dificultades, como lo ha hecho hasta ahora, explica Gnesotto, parece previsible que en veinte años estas tendencias no impongan grandes dificultades a la estabilidad política de la región. En este sentido, dada la preocupación de la UE por la excesiva militarización del conflicto guerrillero y su consideración de este asunto como consecuencia de problemas paralelos como la pobreza (la Estrategia Europea de Seguridad antes comentada describe claramente esta posición), esta región seguirá siendo un aliado para Colombia mientras desee abordar el problema en toda su complejidad.

Precisamente una dificultad para Colombia puede ser la pérdida de importancia en el ámbito público internacional de la guerra contra el tráfico de drogas (en comparación con décadas anteriores), a favor de la preocupación de la comunidad internacional por el calentamiento global, por ejemplo. Bien considerado, el tema medioambiental puede ser un elemento de enganche importante teniendo en cuenta la riqueza natural de Colombia y el hecho de compartir, junto con otros países latinoamericanos, uno de los pulmones más importantes de la tierra: el Amazonas, lo que constituye una fuente de desarrollo, que podría basarse en la iniciativa "Biotrade" de la UNCTAD, por ejemplo. Así aportaría una concepción a la economía, más cercana a la responsabilidad medioambiental, y le permitiría luchar desde la raíz con la producción ilegal de cocaína.

En relación con esto, la creciente especialización de la UE en el sector químico y farmacéutico, (Gnesotto, 2005) unido a la convergencia de las tecnologías nano-bio-info-cogno en el sector salud, pueden hacer de Colombia un importante socio para la UE.
Por otro lado, la UE es el principal socio comercial de América Latina en general y el tercero más importante de Colombia (DANE, 2007) ${ }^{21}$. La creciente necesidad de la UE de adquirir bienes básicos hace de la región latinoamericana y de Colombia, especialmente, un socio atractivo.

Desde el punto de vista internacional, y teniendo en cuenta que el dinamismo económico se concentra cada vez más en el norte de Occidente y en Asia, contar en América Latina con países emergentes como Brasil y México, puede transformarse en un factor impulsor o un freno para el desarrollo de la región. Si América del Sur desea sacar provecho de esta ventaja, tanto para insertarse en el círculo virtuoso del crecimiento brasileño, como para ampliar los puntos de encuentro con la UE, será necesario para los países de la región y para Colombia, en particular, acercarse a este país. Igualmente el entorno colombiano se hace interesante ante la Unión Europea, gracias a las sinergias creadas en el marco de la Comunidad Andina que cuenta con fuentes energéticas importantes y que a futuro puede desarrollar su amplio potencial en energías limpias como biocarburantes (asunto de gran interés para los países desarrollados y emergentes), así como el empuje del crecimiento de Chile para la economía suramericana.

No es fácil para Colombia atender estas oportunidades, teniendo en cuenta que también debe intensificar sus relaciones con Asia y que en términos generales los países latinoamericanos necesitarán dedicarse los próximos años a la creación de infraestructuras notablemente abandonadas en los últimos años a la mejora de su capital humano, del clima de confianza en el país y de su gobernabilidad (Gnesotto, 2005)

\footnotetext{
${ }^{21}$ Ver http://www.dane.gov.co/index.php?option=com_content\&task=category\&sectionid=15\&id= 49\&Itemid=231.
} 
El panorama anterior, deja en evidencia el amplio abanico del que pueden elegir los países en desarrollo para construir asociaciones en diversos ámbitos (comercial, cooperación, político, tecnológico, etc.), así como la creciente importancia de los países emergentes y su mayor vocación exterior que les abrirá las puertas. Esta multipolaridad obliga a los países desarrollados a tener en cuenta a estos nuevos actores de gran relieve en las decisiones importantes del sistema internacional y, por lo tanto, les obliga a esforzarse más. Dicho de otro modo, en la medida que América Latina logre construir alianzas estratégicas con los países emergentes, los países desarrollados se preocuparán por no quedarse detrás, tal como Estados Unidos se preocupa por el creciente número de inversiones estratégicas de China en la región.

La situación presentada hasta estas líneas muestra un panorama de tendencias en el que todo sigue igual, aunque trata de mostrar algunos puntos que pueden dar pie a giros importantes. Lo fundamental es que dadas las tendencias regionales y mundiales, Colombia y, América Latina en general, necesitan formas novedosas para captar la atención de socios que les empujen hacia un escenario más positivo y la clave puede estar en los factores que generan fuertes interdependencias.

\section{Comentarios finales}

La creciente dinámica de la interdependencia ha estado presente a lo largo de todo el análisis. Además de las interrelaciones entre los diversos actores del sistema internacional que se han mostrado, ha quedado claro que también existen fuertes interconexiones entre tendencias que marcan la globalización o la seguridad. Este es sin duda un mundo interdependiente, en el que el predominio de las tendencias positivas puede alcanzar una gran cantidad de espacios en el sistema internacional, pero en el que también la consolidación de las tendencias negativas puede arrastrar al orden internacional hacia un modelo cada vez más anárquico.

El futuro de la UE depende de su eficacia en crear, en diversos ámbitos, alianzas que fortalezcan las tendencias positivas y que logren positivizar algunas tendencias negativas.
Un mundo en el que inevitablemente existirán factores negativos, pero en el que predomine el crecimiento económico mundial en torno a los países desarrollados y emergentes, arrastrando a los países en desarrollo; en el que se promueva la gobernanza global mediante el diálogo entre actores diversos;,en el que se reafirme la democracia y los derechos humanos; en el que el diálogo sobre la seguridad se apoye en el desarrollo y la estabilidad política; en el que el medio ambiente sirva para movilizar fuerzas supranacionales hacia una dirección positiva; en el que se acerque a un equilibrio entre envejecimiento y presiones migratorias, será un mundo que permitirá a la UE dar mayor contenido a sus principios constitutivos (libertad, igualdad, diversidad, solidaridad) tanto interna como internacionalmente y en el que seguirá evolucionando la concepción de la seguridad mundial y de la progresividad de su proyecto. 
Es este el escenario que aportará oportunidades más seguras al futuro modelo europeo, reforzará su carácter cosmopolita y marcará su vocación internacional.

En un entorno con estas características las posibilidades de América Latina y Colombia son claramente positivas. La concepción de la UE en asuntos como el desarrollo, la seguridad o el medioambiente y sus intereses económicos en la región latinoamericana son dos aspectos a los que Colombia debe engancharse para obtener mejores resultados en su agenda.

\section{Bibliografía}

- BAREA, Maite (2003, $2^{\mathrm{a}}$ ed.), En los orígenes e la Unión Europea. Robert Schuman y Jean Monnet. Madrid, Ediciones Encuentro.

- BECK, Ulrich (2006), La Europa Cosmpolita. Sociedad y política en la segunda modernidad. Barcelona, Paidós.

- CAVALIER, Bruno y Nicolas MEUNIER (2006), "L'Europe e toutes ses frontieres", Eclairages, Crédit Agricole, Noviembre, № 105.

- Consejo de la Unión Europea (2003), " Europa segura en un mundo mejor ", Estrategia Europea de Seguridad, 12 e diciembre de 2003.

- Consejo de Lisboa (2007), " Informe Conjunto sobre el empleo 2006/207 ", Bruselas, DG/GII, 6706/07.

- EU (2007), " Los próximos 50 años " : http://europa.eu/50/future/index_es.htm

- Eurobarometer (2006), "European Social Reality", Special Eurobarometer, №273, NoviembreDiciembre.

- Eurobarmeter (2007), "Attitude on issues related to the EU energy policy. Analytical Report", Flash Eurobarometer, 206a

- Eurostat (2007), "Science, Technology and Innovation in Europe", Bruselas, Eurostat, Pocketbooks.

- FONTELA, Emilio (2006), Europa en un mar de dudas, Dinero, junio, № 955.

- FONTELA, Emilio y Marybel PÉREZ (2007), "Las relaciones de China con sus vecinos asiáticos", Madrid, Reunión de Economía Mundial, 18 de abril de 2007.

- GNESOTTO, Nicole (Director), (2005), The new global Puzzle. What World for the EU in 2025?. París, Institute for Security Studies.

- NAïR, S. (1997) Rapport et bilan et d'orientation sur la politique de codeveloppement liée aux flux migratoires. Ministère de Affaires Etrangères.

- Observ'ER (2006), "6me Etat des energies renouvelables en Europe" Observ'ER.

- ORTEGA, Martin (2007), "Building the Future. The EU's contribution to global governance", Chaillot Paper, No 100, París, Institute for Security Studies.

- PARSONS, Craig (2002) "Showing Ideas as Causes: The origins of the European Union", International Organization. Vol. 56, №1, pp 47-84.

- ROSAMOND, Ben (2005), "Conceptualizing the EU Model of Governance in World Politics", European Foreign Affairs Review. 10:463-478. 